\title{
Structure of the $(\sqrt{3} \times \sqrt{3}) R 30^{\circ} \mathrm{Ag} / \mathrm{Si}(111)$ Surface from First-Principles Calculations
}

\author{
Y. G. Ding, C. T. Chan, and K. M. Ho \\ Ames Laboratory-U.S. Department of Energy and Department of Physics, Iowa State University, Ames, Iowa 50011
}

(Received 20 March 1991)

\begin{abstract}
The structure of $(\sqrt{3} \times \sqrt{3}) \mathrm{Ag} / \mathrm{Si}(111)$ is investigated using first-principles total-energy calculations. The lowest-energy configuration consists of a top layer of $\mathrm{Ag}$ atoms arranged as "honeycomb chained trimers" lying above a distorted "missing-top-layer" Si(111) substrate. We find that the honeycomb structure observed in scanning-tunneling-microscope images arises from the electronic charge density of an empty surface band near the Fermi level.
\end{abstract}

PACS numbers: $68.35 . \mathrm{Bs}, 68.35 . \mathrm{Md}, 73.20 . \mathrm{At}$

The atomic geometry of the $(\sqrt{3} \times \sqrt{3})$ structure observed during the adsorption of $\mathrm{Ag}$ on $\mathrm{Si}(111)$ is a problem that has baffled surface scientists for over a decade [1-32]. Many techniques [1-29], including scanning tunneling microscopy (STM), photoemission, photoelectron diffraction, ion scattering, x-ray diffraction, LEED, reflection high-energy electron diffraction (RHEED), and surface extended $\mathrm{x}$-ray-absorption fine-structure spectroscopy, together with some theoretical efforts [30-32], have been used to study the surface. However, the surface structure remains elusive. The main complexity of the problem comes from the large number of plausible and conflicting models that have been proposed for this surface, each model having support from some experiments.

Using first-principles total-energy calculations, we have examined the equilibrium geometries and electronic properties for many plausible models. We found a structural model which not only has the lowest surface energy but can also explain most of the available experimental data on the system. In particular, we find that the honeycomb structure observed in STM images arises not from the top-layer atomic positions but rather from the wavefunction behavior of empty surface electronic states above the Fermi level.

The first-principles calculations are performed within the local-density-functional formalism [33] with the Wigner [34] form of the local exchange-correlation potential, and norm-conserving pseudopotentials [35]. The Bloch wave functions are expanded in a mixed basis set [36] comprised of both plane waves (with a 10.5 Ry cutoff) and numerical orbitals centered at $\mathrm{Ag}$ atomic sites. More technical details can be found elsewhere [37]. A "supercell" geometry (equivalent in thickness to twelve Si layers in the $\langle 111\rangle$ direction) is used and there are six layers of $\mathrm{Si}$ beneath the $\mathrm{Ag}$ layer [38]. If the $\mathrm{Ag}$ layer is in an embedded geometry, there are additional $\mathrm{Si}$ atoms above the Ag layer. Self-consistency is obtained with seven $k$ points in the two-dimensional irreducible Brillouin zone. Forces on the atoms are calculated for each model and the atoms are fully relaxed (laterally and vertically) to their zero-force equilibrium positions within the symmetry constraints imposed by the model. In some cases, multiple starting configurations are tested to minimize the possibility of landing on local rather than global minimum.
The models we have investigated can be divided into three groups: (i) $\frac{2}{3}$-monolayer (ML) Ag coverage models with a top Ag layer arranged in a honeycomb, such as the $\mathrm{Ag}$ honeycomb (HC) model [2,17], and various "missing-top-layer" (MTL) models [9,11,26]; (ii) 1-ML Ag coverage models, with Ag atoms embedded and a top honeycomb layer of $\mathrm{Si}$ (with $\frac{1}{3}$ vacancies), such as the embedded trimer (ET) model [1], the substitutional trimer (ST) model [20], and models of Vlieg et al. [6]; (iii) $1 \mathrm{ML}$ of $\mathrm{Ag}$ atoms on the top layer above all the $\mathrm{Si}$ atoms, such as the HCT-1 and HCT-2 models, and also $(1 \times 1) \mathrm{Ag} / \mathrm{Si}$ models (for comparison purposes). The HCT-1 and HCT-2 are named after the honeycombchained-trimer (HCT) arrangement of the Ag layer proposed from x-ray diffraction [5] and RHEED [26] experiments. The HCT-1 model has a missing-top-layer Si(111) substrate, while the HCT-2 has a full doublelayer Si substrate. For many of the models, different Ag registries are considered so as to locate the one with the lowest energy.

Since there are two possible coverages and hence different stoichiometries, the quantity that governs the relative stability of different models is the surface energy per $(\sqrt{3} \times \sqrt{3})$ unit cell of the composite $\mathrm{Ag} / \mathrm{Si}$ system, which we define to be $\frac{1}{2}\left(E_{\text {tot }}-N_{\mathrm{Ag}} E_{\mathrm{Ag}}-N_{\mathrm{Si}} E_{\mathrm{Si}}\right)$, where $E_{\text {tot }}$ is the total energy of the slab, $N_{\mathrm{Ag}}$ and $N_{\mathrm{Si}}$ are the number of $\mathrm{Ag}$ and $\mathrm{Si}$ atoms in the slab, respectively, and $E_{\mathrm{Ag}}$ and $E_{\mathrm{Si}}$ are the total energy per atom of bulk $\mathrm{Ag}$ and Si. The factor of one-half appears because there are two surfaces in a slab. The HCT-1 model has the lowest surface energy.

The structure of the HCT-1 model is shown schematically in Fig. 1(a) (top view) and Fig. 1(b) (side view). The structure can be envisioned as follows: Start with a clean missing-top-layer $\mathrm{Si}(111)$ surface (i.e., each toplayer atom has three dangling bonds). Put one monolayer of $\mathrm{Ag}$ above the fifth (or sixth) Si layer, so that it is $0.85 \AA$ above the first $\mathrm{Si}$ layer and $3.15 \AA$ above the second Si layer (the first intact double layer). The Ag layer reconstructs, with lateral displacements characterized by a parameter $x$ [see Fig. 1 (a)] which is found to be $0.43 a$ ( $a$ is the lattice parameter of the surface unit cell). After the reconstruction, the $\mathrm{Ag}-\mathrm{Ag}$ has a nearestneighbor (nn) distance of $3.45 \AA$. The in-plane nn Si-Si distance in the top $\mathrm{Si}$ layer is substantially smaller than 
(a)

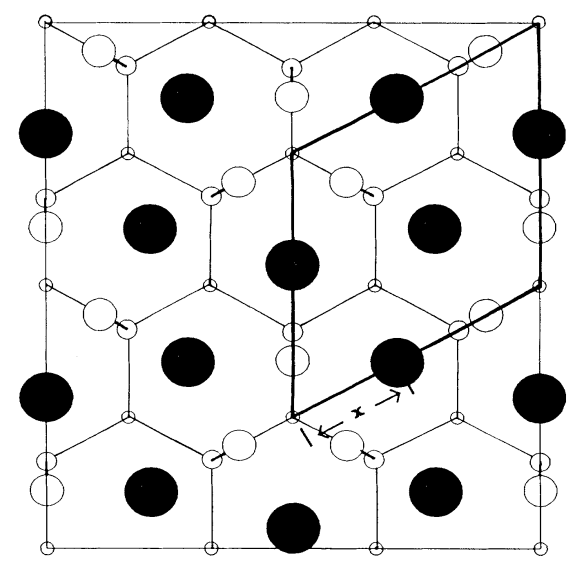

(b)

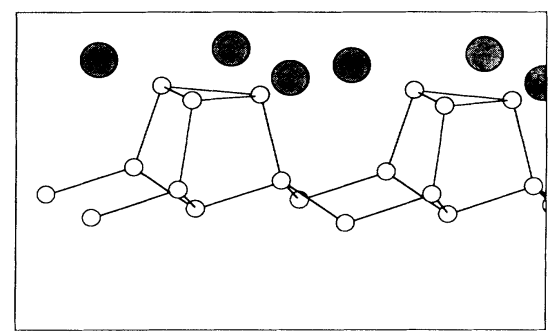

FIG. 1. Geometrical structure of the HCT-1 model: (a) top view; (b) side view. Shaded and empty circles correspond to $\mathrm{Ag}$ and $\mathrm{Si}$, respectively.

that on an ideal surface so that two of the three broken bonds of the top Si layers are reformed by the Si trimers. The remaining dangling bond is satisfied by the $\mathrm{Ag}$ atoms. The nn Ag-Si distance is $2.54 \AA$. The third Si layer has a buckling of about $0.2 \AA$, with $\frac{2}{3}$ of the atoms positioned above the other $\frac{1}{3}$. Deeper-layer relaxations

TABLE I. Comparison of the theoretical structural parameters of the lowest-energy HCT-1 model with RHEED [26], $x-$ ray diffraction $[5,6]$, and $x$-ray standing-wave [7] results. The $z-\mathrm{Ag}$ and $z-\mathrm{Si}$ are the vertical distances of the top Ag and MTL Si layers above the first Si double layer.

\begin{tabular}{llllc}
\hline \hline & & & & \\
& RHEED $^{\mathrm{a}}$ & \multicolumn{1}{c}{$\mathrm{X} \mathrm{ray}^{\mathrm{b}}$} & \multicolumn{1}{c}{$\mathrm{X} \mathrm{ray}^{\mathrm{c}}$} & $\begin{array}{c}\text { Theory } \\
(\text { HCT }-1)\end{array}$ \\
\hline$x / a$ & 0.46 & $0.44 \pm 0.15$ & $0.43 \pm 0.04$ & 0.43 \\
$\mathrm{Ag}-\mathrm{Ag}(\AA)$ & 3.36 & 3.39 & 3.43 & 3.45 \\
$\mathrm{Ag}-\mathrm{Si}(\AA)$ & & 2.54 & $2.57 \pm 0.05$ & 2.54 \\
& & 2.68 & $2.63 \pm 0.06$ & 2.60 \\
$\mathrm{Si}-\mathrm{Si}(\AA)$ & $2.7 \pm 0.5$ & 2.30 & $2.32 \pm 0.04$ & 2.51 \\
$z-\mathrm{Ag}(\AA)$ & $2.95 \pm 0.05$ & $2.95 \pm 0.1$ & $3.05 \pm 0.02^{\mathrm{d}}$ & 3.15 \\
$z-\mathrm{Si}(\AA)$ & $2.2 \pm 0.1$ & $2.1 \pm 0.2$ & $2.26^{\mathrm{e}}$ & 2.30 \\
\hline \hline
\end{tabular}

a Reference [26].

${ }^{\mathrm{b}}$ Reference [5].

${ }^{c}$ Reference [6].

${ }^{\mathrm{d}} \mathrm{x}$-ray standing wave [7].

${ }^{e} \mathrm{E}$. Vlieg (private communication).

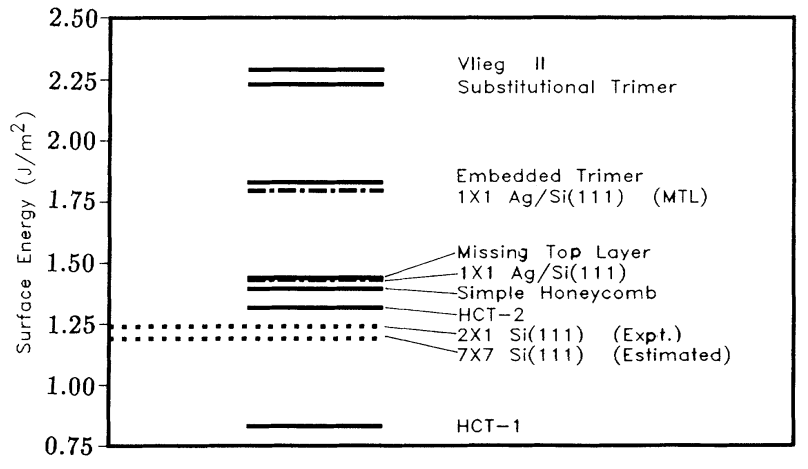

FIG. 2. Comparison of the surface energies for various models. Experimental surface energy for $(2 \times 1) \mathrm{Si}(111)$ is quoted from Ref. [44], and the surface energy of $(7 \times 7) \mathrm{Si}(111)$ is taken to be $0.04 \mathrm{eV} /$ (surface atom) lower than the $(2 \times 1)$ surface [45]. The $(1 \times 1) \mathrm{Ag} / \mathrm{Si}(111)$ marked with (MTL) has a "missing-top-layer" $\mathrm{Si}$ substrate while the $(1 \times 1) \mathrm{Ag} / \mathrm{Si}(111)$ (unmarked) has an intact double-layer Si substrate.

have to be tested with thicker-slab calculations. We note that if we impose a $(1 \times 1)$ surface unit cell (lateral relaxations not allowed), $1 \mathrm{ML}$ of $\mathrm{Ag}$ on top of an intact double-layer Si surface actually has a lower surface energy than the one with a MTL configuration. It is the substantial reconstruction of the top-layer $\mathrm{Si}$ atoms allowed in the MTL configuration that makes it more favorable in the $(\sqrt{3} \times \sqrt{3})$ unit cell.

Several more recent experimental investigations [39, 40] have proposed models essentially identical to the HCT-1 structure. Model II of Vlieg et al. [6] is also equivalent to HCT-1 if we discard the top Si honeycomb layer, and the model of Ichimiya et al. [26] is similar to HCT-1 except for an extra twisting of the Si trimers. Our structural parameters are compared with those reported in RHEED and x-ray experiments in Table $\mathrm{I}$.

The surface energy of the Ag/Si system for a number of models at their respective lowest-energy configurations are compared in Fig. 2. We found that all the models in the second group ( $1 \mathrm{ML}$ embedded $\mathrm{Ag}$ ) tend to have much higher energies than models in the other groups due to the large number of $\mathrm{Si}$ bonds broken to make the top $\mathrm{Si}$ honeycomb layer. Some of the models (e.g., model III in Ref. [6]) have energies so high that they are not shown in Fig. 2. The ET model has the lowest energy in this group, while model II in Ref. [6] has approximately the same energy as ST. We note that the HCT-1 model not only has a lower surface energy than all other competing models, but it is the only model tested that has a surface energy lower than the reconstructed clean $\mathrm{Si}(111)$ surfaces. This indicates that the $\mathrm{Ag}$ atoms should wet the $\mathrm{Si}$ surface rather than display three-dimensional island growth on the surface, consistent with the observed Stranski-Krastanov growth mode of $\mathrm{Ag}$ layers on this surface.

Since the atoms in the top Ag layer are not arranged in 


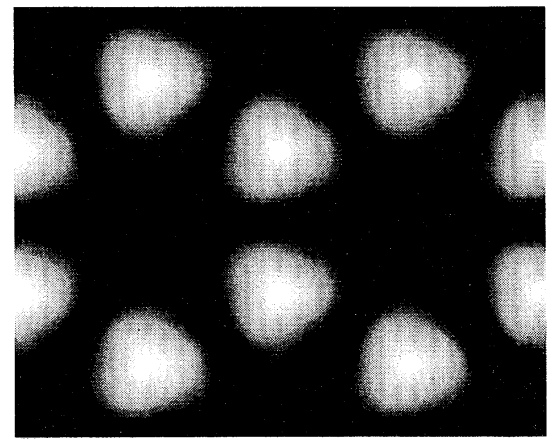

FIG. 3. Electronic charge-density plot for unoccupied surface states on a horizontal plane $1.5 \AA$ above the top $\mathrm{Ag}$ layer. Note the honeycomb arrangement.

a honeycomb structure, our model has to be reconciled with the observed STM images. Since the STM images are governed by the spatial distribution of electronic states involved in the tunneling process [41], we examined the wave functions of electronic states in the energy range from -2.5 to $2.0 \mathrm{eV}$ around the Fermi level $\left(E_{F}=0 \mathrm{eV}\right)$. We found that the charge distributions for the occupied states $(-2.5-0 \mathrm{eV})$ resemble the atomic honeycombchained-trimer arrangement. Above the Fermi level, from 0 to $1 \mathrm{eV}$, the electron charge distribution is dominated by a band of empty surface states. Figure 3 shows a charge-density plot of the states from 0 to $1 \mathrm{eV}$ in a plane parallel to the surface $1.5 \AA$ above the top Ag layer. The plots exhibit the honeycomb pattern observed by STM. This observation is consistent with the bias-voltage dependence of the STM images: The best resolved honeycomb images were obtained for bias conditions where the electrons are tunneling from the tip into the empty surface states. We also observe from Fig. 3 that the maxima of the electronic distribution for the empty states occur at the center of the Ag trimers, and are situated over the fourth $\mathrm{Si}$ layer, in agreement with the registry determined by Wilson and Chiang [3].

Figure 4 shows the electronic density of states (DOS) of the slab for the HCT-1 model [42]. We notice that $E_{F}$ is located near the bottom of a "pseudogap": a region of about $1 \mathrm{eV}$ where the DOS drops to a very low value. The states in this energy region come from a surface band with charge localized mainly on the Ag layer. This band overlaps with the valence-band top at the center of the surface Brillouin zone by less than $\sim 0.1 \mathrm{eV}$, and then rises sharply with increasing $\mathbf{k}$ away from the zone center giving rise to a very low DOS. HCT-1 is the only model we have studied that gives something close to a gap in the DOS near $E_{F}$. Experimentally, photoemission experiments have reported a gap for the $(\sqrt{3} \times \sqrt{3})$ surface. It is possible that the small density of states in the pseudogap region can escape detection in these experiments. However, surface charging effects may also be important for the interpretation of the photoemission data. Quanti-

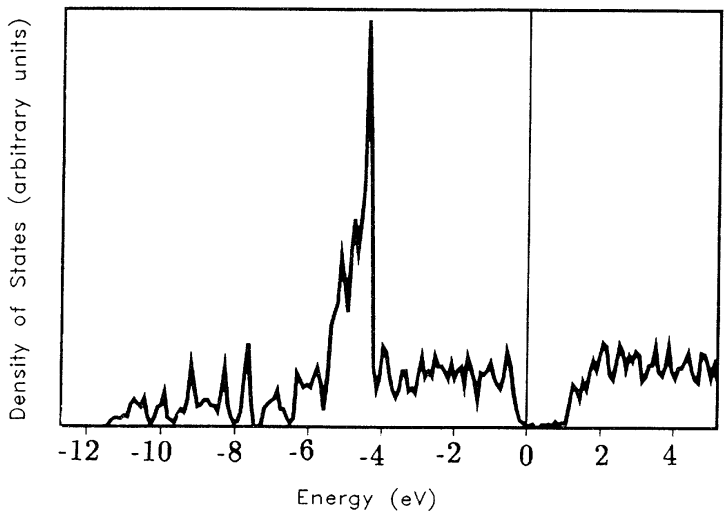

FIG. 4. Density of states for the HCT-1 model.

tative comparison between our results and photoemission and STM experiments will have to include the effects of surface charging [11] and surface band bending, as well as the shift of energy position of the surface band relative to the bulk states as the surface becomes charged [43].

We thank Dr. E. Vlieg for providing the third column of Table I. The work was supported by the National Science Foundation (Grant No. DMR-8819379), and in part by the Director for Energy Research, Office of Basic Energy Sciences, including a grant of computer time on the Cray computers at Lawrence Livermore Laboratory. The Ames Laboratory is operated for the U.S. Department of Energy by the Iowa State University under Contract No. W-7405-Eng-82.

[1] E. J. van Loenen, J. E. Demuth, R. M. Tromp, and R. J. Hamers, Phys. Rev. Lett. 58, 373 (1987).

[2] R. J. Wilson and S. Chiang, Phys. Rev. Lett. 58, 369 (1987).

[3] R. J. Wilson and S. Chiang, Phys. Rev. Lett. 59, 2329 (1987).

[4] J. M. Nicholls, F. Salvan, and B. Reihl, Phys. Rev. B 34, 2945 (1986).

[5] T. Takahashi, S. Nakatani, N. Okamoto, T. Ishikawa, and S. Kikuta, Jpn. J. Appl. Phys. 27, L753 (1988).

[6] E. Vlieg, A. W. Denier van der Gon, J. F. van der Veen, J. E. MacDonald, and C. Norris, Surf. Sci. 209, 100 (1989).

[7] E. Vlieg, E. Fontes, and J. K. Patel, Phys. Rev. B 43, 7185 (1991).

[8] S. Kono, K. Higashiyama, and T. Sagawa, Surf. Sci. 165, 21 (1986).

[9] E. L. Bullock, G. S. Herman, M. Yamada, D. J. Friedman, and C. S. Fadley, Phys. Rev. B 41, 1703 (1990).

[10] S. Kono, T. Abukawa, N. Nakamura, and K. Anno, Jpn. J. Appl. Phys. 28, L1278 (1989).

[11] S. Kono, K. Higashiyama, T. Kinoshita, T. Miyahara, H. Kato, H. Ohsawa, Y. Enta, F. Maeda, and Y. Yaegashi, Phys. Rev. Lett. 58, 1555 (1987). 
[12] G. Lelay, Surf. Sci. 132, 169 (1983).

[13] T. Yokotsuka, S. Kono, S. Suzuki, and T. Sagawa, Surf. Sci. 127, 35 (1983).

[14] Y. Horio and A. Ichimiya, Surf. Sci. 164, 589 (1985).

[15] M. Copel and R. M. Tromp, Phys. Rev. B 39, 12688 (1989).

[16] M. Watamori, F. Shoji, T. Hanawa, and K. Oura, Surf. Sci. 226, 77 (1990); K. Oura, M. Watamori, F. Shoji, and T. Hanawa, Phys. Rev. B 38, 10146 (1988).

[17] M. Aono, R. Souda, C. Oshima, and Y. Ishizawa, Surf. Sci. 168, 713 (1986).

[18] M. Saitoh, F. Shoji, K. Oura, and T. Hanawa, Surf. Sci. 112, 306 (1981).

[19] R. S. Williams, R. S. Daley, J. H. Huang, and R. M. Charatan, Appl. Surf. Sci. 41/42, 70 (1989).

[20] T. L. Porter, C. S. Chang, and I. S. T. Tsong, Phys. Rev. Lett. 60, 1739 (1988).

[21] K. Sumitomo, K. Tanaka, Y. Izawa, I. Katayama, F. Shoji, K. Oura, and T. Hanawa, Appl. Surf. Sci. 41/42, 112 (1989).

[22] W. C. Fan, A. Ignatiev, H. Huang, and S. Y. Tong, Phys. Rev. Lett. 62, 1516 (1989).

[23] F. Wehking, H. Beckermann, and R. Niedermayer, Surf. Sci. 71, 364 (1978).

[24] Y. Terada, T. Yoshizuka, K. Oura, and T. Hanawa, Surf. Sci. 114, 65 (1982).

[25] Y. Horio and A. Ichimiya, Surf. Sci. 133, 393 (1983).

[26] A. Ichimiya, S. Kohmoto, T. Fujii, and Y. Horio, Appl. Surf. Sci. 41/42, 82 (1989).

[27] S. Hasegawa, H. Daimon, and S. Ino, Surf. Sci. 186, 138 (1987).

[28] J. Stohr and R. Jaeger, Surf. Sci. 134, 813 (1983).

[29] M. Hanbucken, M. Futamoto, and J. A. Venables, Surf. Sci. 147, 433 (1984).

[30] S. H. Chou, A. J. Freeman, S. Grigoras, T. M. Gentle, B. Delley, and E. Wimmer, J. Chem. Phys. 89, 5177 (1988).
[31] Q. Q. Zheng and Z. Zeng, Surf. Sci. 195, L173 (1988).

[32] C. T. Chan and K. M. Ho, Surf. Sci. 217, 403 (1989).

[33] See, e.g., Theory of the Inhomogeneous Electron Gas, edited by N. H. March and S. Lundqvist (Plenum, New York, 1983).

[34] E. Wigner, Phys. Rev. 46, 1002 (1934).

[35] D. R. Hamann, M. Schluter, and C. Chiang, Phys. Rev. Lett. 43, 1494 (1979).

[36] S. G. Louie, K. M. Ho, and M. L. Cohen, Phys. Rev. B 19, 1774 (1979).

[37] N. Takeuchi, C. T. Chan, and K. M. Ho, Phys. Rev. B 40, 1565 (1989).

[38] By comparing the results of the same model calculated with six-layer and eight-layer $\mathrm{Si}$ slabs (both fully relaxed), the error in surface energy due to finite slab thickness is estimated to be about $0.1 \mathrm{~J} / \mathrm{m}^{2}$.

[39] T. Takahashi, S. Nakatani, N. Okamoto, T. Ishikawa, and S. Kikuta, Surf. Sci. 242, 54 (1991).

[40] M. Katayama, R. S. Williams, M. Kato, E. Nomura, and M. Aono, Phys. Rev. Lett. 66, 2762 (1991).

[41] J. Tersoff and D. R. Hamann, Phys. Rev. Lett. 50, 1998 (1983).

[42] The slab densities of states (SDOS) are obtained from the eigenvalues of 37 evenly spaced $k$ points in the irreducible Brillouin zone with a Gaussian smearing of 0.2 eV. SDOS computed with $70 k$ points show little changes.

[43] K. M. Ho, C. L. Fu, D. M. Kolb, and G. Piazza, J. Electroanal. Chem. 150, 235 (1983); D. M. Kolb, W. Boeck, K. M. Ho, and S. H. Liu, Phys. Rev. Lett. 47, 1921 (1981).

[44] J. A. Venables, J. S. Drucker, M. Krishnamurthy, G. Raynerd, and T. Doust, Mater. Res. Soc. Symp. Proc. 198, 93 (1990).

[45] Guo-Xin Qian and D. J. Chadi, Phys. Rev. B 35, 1288 (1987). 


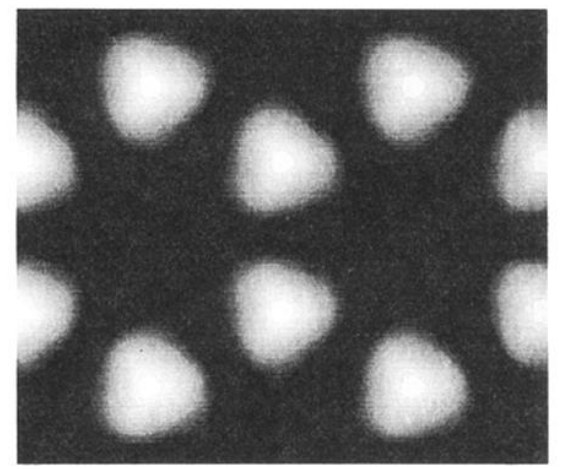

FIG. 3. Electronic charge-density plot for unoccupied surface states on a horizontal plane $1.5 \AA$ above the top $\mathrm{Ag}$ layer. Note the honeycomb arrangement. 